\title{
Neural similarity between mentalizing and live social interaction
}

$$
\text { Junaid S Merchant }{ }^{1,2} \text {, Diana Alkire }{ }^{1,2}, \text { \& Elizabeth Redcay }{ }^{1,2}
$$

${ }^{1}$ Neuroscience and Cognitive Science Program, University of Maryland, College Park

${ }^{2}$ Department of Psychology, University of Maryland, College Park 


\begin{abstract}
Reciprocal social interactions are a quintessential part of human life, yet little neuroimaging research has examined the underlying neurocognitive mechanisms using social interactive experimental paradigms. Recent work using social interactive tasks has demonstrated brain activity in the mentalizing network, even in the absence of explicit mentalizing demands, suggesting that social interactive contexts automatically engage mentalizing. However, while overlapping brain activations are suggestive of similar underlying neural processes between explicit mentalizing and spontaneous mentalizing during interaction, they are not a direct test of whether or not these processes are represented similarly in the brain. Pattern-based approaches provide the sensitivity to examine the similarity between different neurocognitive processes. The current study used representational similarity analysis on a task wherein participants made mental and non-mental judgments about an abstract character and a live, social interactive partner during fMRI. The within-subject, 2 (Mental/Non-mental) $\times 2$ (Peer/Character) design enabled us to examine the similarity in response patterns between conditions across numerous brain regions associated with social cognition, and estimate fit to three theoretical models of how the two processes relate: 1) social interaction and explicit mentalizing about an abstract character are represented similarly; 2) interactive peer and abstract character are represented differently regardless of the evaluation type; and 3) mental and non-mental states are represented dissimilarly regardless of target. Results demonstrate that the temporal poles and the right posterior superior temporal sulcus represent mentalizing and peer interactions similarly (Model 1), suggesting that response patterns in these regions provide a link between mentalizing and social interaction. Much of the rest of the social brain exhibits different representations for interactive peers and abstract characters (Model 2). Finally, model-free analyses showed that different regions show sensitivity to either the interaction partner or story character. Together, our findings highlight the importance of studying social cognitive processes using interactive approaches, and the utility of pattern-based approaches in understanding how social cognitive processes relate to each other.
\end{abstract}




\section{Introduction}

Social interactions, or reciprocal exchanges between socially engaged individuals, are a ubiquitous aspect of daily life and play an important role in shaping the human brain. Despite the relative ease with which most individuals are able to engage in social interactions, a myriad of neurocognitive processes underlie this complex social behavior. Mentalizing, or the ability to infer the mental state of others $(1,2)$, is thought to be critical for navigating social interactions. Neuroimaging investigations of mentalizing over the past twenty years have revealed a common network of brain regions that are activated across a range of tasks that require mental state reasoning, including dorsal and ventral regions of the medial prefrontal cortex (dmPFC and vmPFC), inferior frontal gyrus (IFG), dorsolateral prefrontal cortex (dIPFC), precuneus, temporoparietal junction (TPJ), anterior temporal lobe (ATL), and superior temporal sulcus (STS; 3-5). However, most neuroimaging investigations of mentalizing have utilized non-interactive experimental paradigms wherein individuals make third-person attributions about abstract characters or simply observe social behavior. Thus, relatively little is known about the role of mentalizing when an individual is actively engaged in a social interaction.

Neuroimaging studies utilizing paradigms that involve engaging with a social partner in real time - a set of approaches collectively referred to as "second-person neuroscience" - have started to shed light on the neurocognitive processes involved in social interaction $(6,7)$. This line of work has revealed that engaging with a live social

partner recruits a more extended network of brain systems than has been previously reported in studies relying on non-interactive, third-person approaches, which includes 
the mentalizing, reward, affective, and mirror neuron brain networks (7-10). The distinction in brain activations between traditional third-person and newer second-person approaches highlights the difference in "offline" and "online" social cognition - terms that signify the difference between the cognitive processes involved in thinking about another who is separated in time and space from the self, and the cognitive processes engaged by the dynamical nature of live, reciprocal interactions, respectively. Interestingly, second-person approaches have revealed activity in the mentalizing network when an individual simply engages with a live social partner, even in the absence of explicit mentalizing demands $(7,9,11)$. These findings suggest that, rather than a top-down process, mentalizing may be spontaneously elicited in social-interactive contexts. However, activations in the mentalizing network do not necessarily indicate the engagement of the mentalizing process.

All of the brain regions comprising the "mentalizing network" have been implicated in numerous other higher-order functions across cognitive contexts, so reversely inferring the mentalizing process based on the spatial location of brain activity is particularly error-prone $(12,13)$. Thus, it is unknown if social interaction engages spontaneous mentalizing, if the two simply elicit activity in overlapping swaths of brain tissue despite subserving dissociable functions, or if there is a different cognitive process involved in both social interaction and mentalizing. For instance, Alkire and colleagues (11) reported overlapping activations in the ATL, STS, and IFG when participants engaged in social interactions that required no mental state reasoning and when participants were asked to make inferences based on the mental state of an abstract character, suggesting that social interaction induces spontaneous mentalizing. 
However, these regions are also associated with other functions that may play a role in social interaction. For example, the ATL and STS are also associated with the representation of person-specific information (14-21), while the IFG has been implicated in a range of executive processes, such as working memory and behavioral inhibition (22-24). Thus, it is unclear if the overlapping activity associated with social interaction reported by Alkire et al. (11) is specific to mentalizing or reflects other higher-order cognitive processes.

Multi-voxel pattern analysis (MVPA) focuses on the information represented in distributed patterns of brain activity, and helps alleviate some of the problems of reverse inference used in traditional activation studies $(25,26)$. In particular, representational similarity analysis (RSA), a specific form of MVPA, leverages voxel-wise activation patterns to quantify the similarity between neural responses elicited by different task conditions as a means to infer their neurocognitive similarity, and thereby enables the testing of competing cognitive models (27). For example, RSA has proven to be a useful tool for assessing the similarity between domain-general and social-cognitive functions (e.g., similar representations for social, spatial, and temporal distance in the right inferior parietal lobule; 28), and has helped disentangle subtle differences in the neural representations maintained by different brain regions for a common social-cognitive task (e.g., different trait dimensions represented across cortical midline structures activated during self-other judgements; 29). Of interest, applications of this approach have demonstrated that mentalizing regions not only maintain stable representations of mental states across mentalizing targets (i.e., personally known versus abstract others; 30), but also discriminate between socio-emotional features of stories about abstract 
characters $(31,32)$ and contribute to higher-level understanding about the social connections between individuals (33). Moreover, evidence suggests that these regions show increasing discrimination of mental, social, and physical characteristics about an abstract character as children grow older (31). Together, these studies demonstrate that mentalizing regions contribute to the representation of other socio-emotional features, which mature with age, and that this can be uncovered through RSA.

RSA thus provides a framework for examining the similarity in brain response patterns between social interaction and mentalizing as a means to infer similarity in the underlying neurocognitive representations. That is, if the pattern of brain activity that is associated with social interaction in the absence of explicit mentalizing demands is similar to the pattern elicited by mentalizing about an abstract character in a given brain region, this provides additional support for the assumption that social interactions elicit spontaneous mentalizing. However, if the brain region represents person-specific knowledge, then we would expect dissimilar patterns of brain activations for a social interaction partner and an abstract character, regardless of the mentalizing demands. A third possibility is that a brain region's response patterns are specific to explicit mental state reasoning, in which case we expect pattern dissimilarity between mental and non-mental state reasoning, regardless of target. Moreover, examining the similarity of activation patterns across brain regions can provide model-free insights about how different regions cluster or segregate in their representational content, even if they do not fit an a priori model. For example, if the response patterns across conditions for a set of brain regions are correlated, we can think of them as being "representationally connected", and can cluster brain regions based on their similarity structure to derive 
representational connectivity networks $(27,34)$. Model free assessments of the raw neural response patterns can further uncover unexpected similarity structures across conditions. For instance, it is possible that the response patterns in a brain region are sensitive to information about an immediate interaction partner, such that they distinguish mental and non-mental assessments about the partner, but make no such distinctions when making assessments outside of an interaction context.

\section{Current Study}

To understand the neuro-representational relationship between social interaction and mentalizing, the current study implemented RSA on data from a larger sample of participants who underwent the experimental paradigm reported by Alkire and colleagues (11). Participants played a game wherein they alternated between making guesses about a peer who provided them with hints and making guesses about an abstract character, the hints for which were generated by a computer. In both target conditions, half of the hints required using mental state reasoning and half required non-mental, physical information about the target to determine the right answer. This yields a within-subject, 2 (Peer/Character) $\times 2$ (Mental/Non-mental; Figure 1) design to test our three proposed models of how brain representation of social interaction and mentalizing relate to each other (described below), as well as explore model free brain representations associated with each of these processes. To this end, we extracted brain response patterns associated with each of the four conditions, estimated the level of dis/similarity between each pair of conditions using euclidean distance, and assessed how well they fit with three conceptual models of the relationship between conditions. 
A. Task schematic

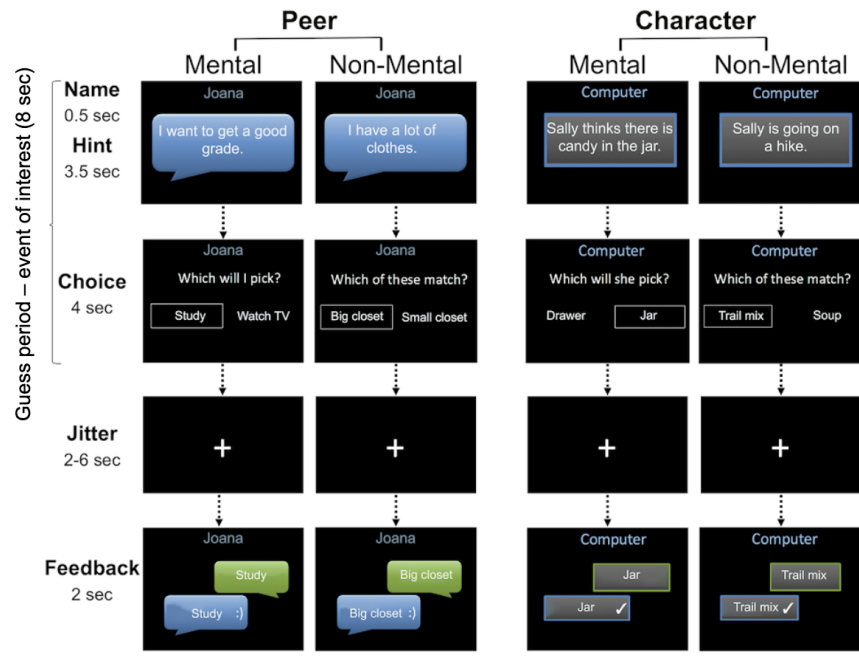

B. 2 × 2 Experimental design

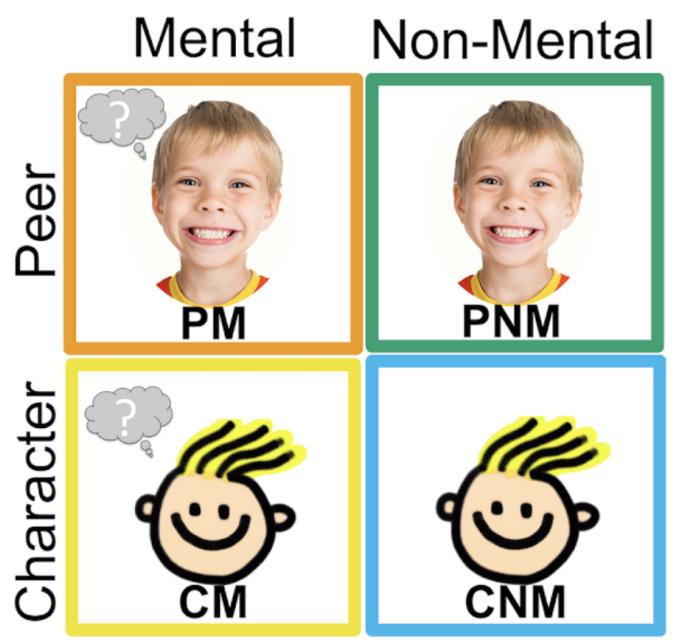

Figure 1. Task schematic (a) and experimental design (b).

Consistent with the idea that social interaction elicits spontaneous mentalizing, Model 1 assumes that all interactions with a peer, regardless of mental state demands, elicit similar neural representations as thinking about the mental state of a character, which are dissimilar from the neural representations elicited when thinking about non-mental characteristics of a character. To determine if a region represents individuating, person-specific knowledge, Model 2 assumes that peer interactions and thinking about characters elicit dissimilar neural responses, but does not distinguish mental and non-mental reasoning for each target. Finally, to assess whether a region is sensitive to mental state representations, Model 3 assumes dissimilar response patterns for mental and non-mental reasoning, but does not distinguish target (Figure 2). 
We calculated the fit of these models first in the regions of interest (ROIs) showing overlapping activations for social interaction and mentalizing reported by Alkire and colleagues (11) to assess if the response patterns supported the authors' interpretation that social interaction elicits mentalizing. To determine the generalizability of representations maintained in brain areas associated with social interaction, we assessed model fit in a set of independent "social interaction" ROls obtained through NeuroSynth (35). Given prior work demonstrating changes in neural representations of mentalizing with age (31), we evaluated model fit as a function of age within these independent ROls. To uncover brain regions showing model fits that were not predicted a priori, we performed searchlight analyses for each model. Finally, to explore if a broader set of "social brain" areas exhibit reliable representations that contribute to social interaction and mentalizing that may differ from our a priori models, we conducted a set of model-free analyses using ROls obtained from Alcalá-López and colleagues (36). The model-free analysis approach we used, which is also known as "representational connectivity", involves estimating the dis/similarity between brain regions, rather than dis/similarity with a priori model, and thereby enables us to examine both the relationships between regions in terms of their response patterns, and evaluate raw brain response patterns to uncover similarity structures that may be different from any of our three models (e.g., distinct response patterns only for peer mental and peer non-mental conditions, but all other conditions are similar). 
A. Condition-wise brain response patterns
B. Neural (dis-)similarity between conditions
C. Fit with conceptual models

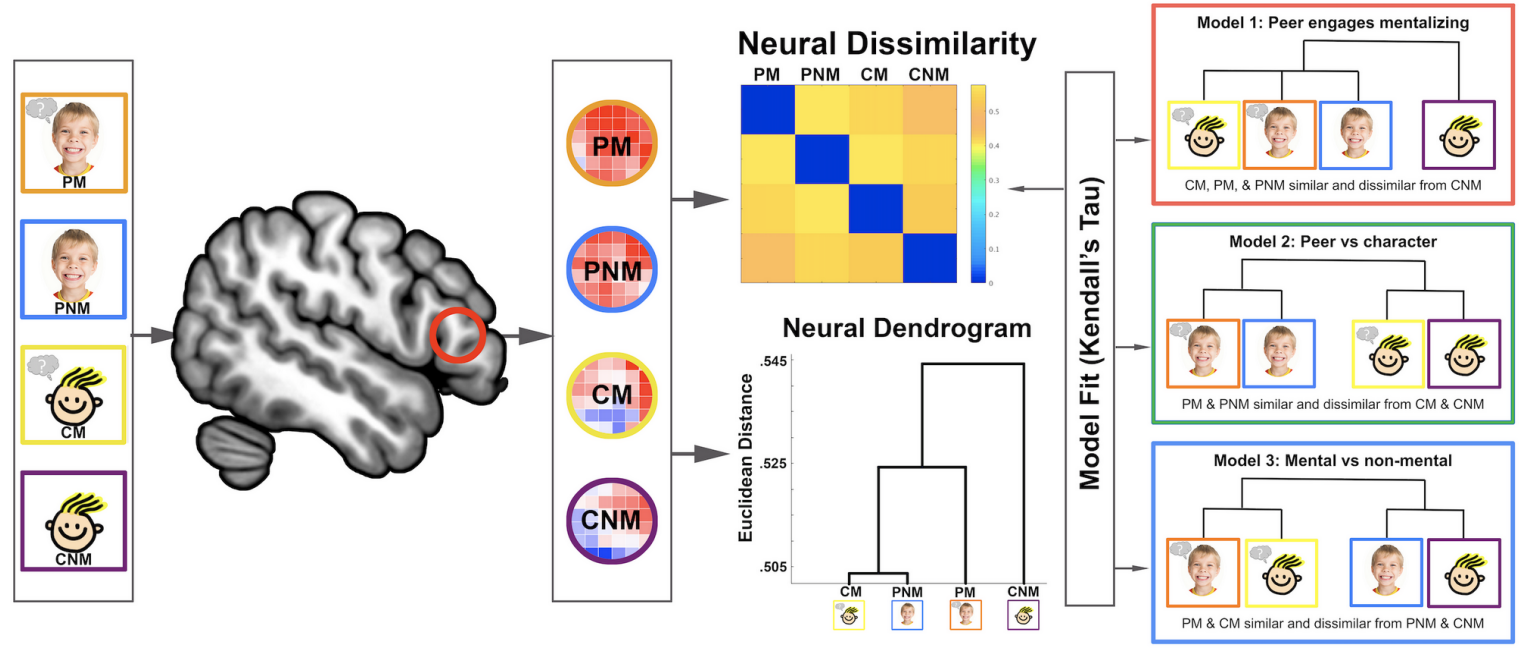

Figure 2. Steps for how RSA works. We first estimated the unsmoothed response pattern for each condition, and extracted the voxel-level response from each ROI (a). The Euclidean distance between each pair of conditions' response patterns were calculated, which can be visualized as a dissimilarity matrix and dendrogram (b). Model fit between an ROI neural dissimilarity and each of our 3 models were estimated using Kendall's Tau.

\section{Methods}

\section{Participants}

A sample of 92 neurotypical 8-14-year-old participants were enrolled in a larger, multi-session project investigating the neural correlates of social interaction during middle childhood. Participants were recruited from the greater Washington, DC area, and exclusionary criteria included MRI contraindications, diagnosis of neurological or psychiatric disorders, first-degree relatives with autism or schizophrenia, and non-native English speakers. A subset of 72 participants were selected for the current analyses because they completed at least two usable fMRI runs of the social interaction fMRI task described in Alkire et al., (11) and believed in the manipulation that they were interacting with another peer in real time. A run was deemed usable if the average head 
motion during the run was under 0.5 millimeter $(\mathrm{mm})$ framewise displacement $(F D)$ as defined by Power et al., (37), and if less than $10 \%$ of the volumes were censored (i.e., regressed out) for having movement over $1 \mathrm{~mm}$ framewise displacement. Of the final sample of 72 participants, 28 were female and 44 were male, with a mean (standard deviation) age of 10.8 (1.79), and an age range of 8-14.6 years. Twenty-eight of these participants are the original sample reported in Alkire et al., $(11 ; 14$ females, mean age $=10.41, S D=1.46$, range $=8.18-12.98)$, and 44 were new participants (14 female, mean age=11.06, $S D=1.94$, range=8-14.6; no significant age or gender differences between sub-samples; Table 1).

\begin{tabular}{|c|c|c|c|}
\hline & $\begin{array}{l}\text { Original Sample } \\
\qquad(\mathrm{N}=\mathbf{2 8})\end{array}$ & $\begin{array}{l}\text { New Sample } \\
\quad(N=44)\end{array}$ & $\begin{array}{l}\text { Full Sample } \\
\qquad(\mathrm{N}=72)\end{array}$ \\
\hline Mean (SD) Age & $10.4(1.46)$ & $11.1(1.94)$ & $10.8(1.79)$ \\
\hline Age Range & $8.18-12.98$ & 8-14.63 & $8-14.63$ \\
\hline Count (\%) Female & $14(50 \%)$ & $14(31.8 \%)$ & $28(38.9 \%)$ \\
\hline $\begin{array}{r}\text { Count (\%) } \\
\text { White/Caucasian }\end{array}$ & $18(64.3 \%)$ & $21(47.7 \%)$ & 39 (54.2\%) \\
\hline $\begin{array}{l}\text { Count (\%) Black/ } \\
\text { African American }\end{array}$ & $9(32.1 \%)$ & $16(36.4 \%)$ & $25(34.7 \%)$ \\
\hline $\begin{array}{r}\text { Count (\%) Latin/ } \\
\text { Hispanic }\end{array}$ & $1(3.6 . \%)$ & $5(11.4 \%)$ & $6(6.9 \%)$ \\
\hline Count (\%) Asian & $0(0 \%)$ & $2(4.5 \%)$ & $2(2.8 \%)$ \\
\hline
\end{tabular}

Table 1. Sample demographic information.

\section{Experimental Protocol}

The experimental protocol is the same as outlined by Alkire and colleagues (11). Briefly, participants were instructed that they would be chatting with a peer in a different lab 
who would also be having their brain scanned, and were shown pictures of two ageand gender-matched peers that they could choose from. In actuality, there were no live interaction peers. Participants were instructed on the "guessing game" that they played during the scan wherein participants were given a hint either by their interaction partner on half the trials (Peer condition), or about a fictional story character on half the trials (Character condition). It was the job of the participant to answer the question "Which will I/she/he pick?" (Mental condition) or "Which of these match?" (Non-mental condition) via button-press to select the appropriate response from the two answer choices. This yielded a fully within-subject, 2 (Peer/Character) $\times 2$ (Mental/Non-mental) design. The 8-second guess phase of each trial was modeled as our epoch of interest and was followed by a 2-6 second jittered period before feedback about the correct answer was provided for 2 seconds. The task was presented using PsychoPy over 4 functional runs, each with 6 trials per condition for a total of 24 trials per run (Figure 1).

\section{Behavioral Data Analysis}

Analyses of behavioral task performance - accuracy (percent correct) and reaction time (RT) in seconds - were conducted using R (38) and JASP (39). Between-group $t$-tests for each condition's accuracy and RT were calculated to assess gender effects, and if either measure showed significant gender differences for any condition, gender was entered as a covariate in the subsequent analysis of variance for the measure. Correlations with age for each condition's accuracy and RT were calculated to assess age effects, and if either measure showed a significant correlation with age for any condition, age was entered as a covariate in subsequent analysis of variance for the 
measure. Accuracy and RT were each entered into two-way repeated measures analysis of variance (with appropriate covariate as needed) to determine main effects of social interaction (Peer vs. Character) and mentalizing (Mental vs. Non-mental), and their interaction. Significant results were interrogated further with follow-up $t$-tests.

\section{MRI Acquisition and Data Processing}

FMRI data were acquired at the Maryland Neuroimaging Center on a 3.0 Tesla scanner with a 32-channel head coil (MAGNETOM Trio Tim System, Siemens Medical Solutions). Four runs of the task were acquired using multiband-accelerated echo-planar imaging (66 interleaved axial slices, multiband factor=6, voxel size $=2.19 \times$ $2.19 \times 2.20 \mathrm{~mm}$, repetition time $=1,250 \mathrm{~ms}$, echo time $=39.4 \mathrm{~ms}$, flip angle $=90^{\circ}$, pixel matrix $=96 \times 96)$ followed by a structural scan (3D T1 magnetization-prepared rapid gradient-echo sequence, 192 contiguous sagittal slices, voxel size $=0.45 \times 0.45 \times 0.90$ $\mathrm{mm}$, repetition time $=1,900 \mathrm{~ms}$, echo time $=2.32 \mathrm{~ms}$, flip angle $=9^{\circ}$, pixel matrix $=512 \times$ 512), and opposite phase-encoding fieldmap scans (66 interleaved axial slices, voxel size $=2.19 \times 2.19 \times 2.20 \mathrm{~mm}$, repetition time $=7,930 \mathrm{~ms}$, echo time=73 ms, flip angle $=90^{\circ}$, pixel matrix $\left.=96 \times 96\right)$. Neuroimaging data were preprocessed using fMRIPrep 1.4.1 (40). Briefly, anatomical images were segmented and normalized to MNI space; functional images were skull-stripped, susceptibility distortion corrected, realigned, slice-time corrected, coregistered and warped to the normalized anatomical image (see https://osf.io/hv3yp/ for full report of the preprocessing pipeline). Subject-level, voxel-wise multiple linear regression was calculated using AFNI's (41) 3dREMLfit for each run separately (to facilitate other, run-level analyses). The guess 
periods for each of the four conditions (i.e., Peer Mental, Peer Non-mental, Character Mental, and Character Non-mental) were convolved with the canonical hemodynamic response using a duration modulated response function (AFNl's dmBlock) with RT as duration to ensure that only the cognition related to the guess period was captured. The feedback period was modeled separately as events of no interest, along with the six motion parameters ( $x, y, z$, roll, pitch, and yaw), their derivatives, and volumes censored due to FD $>1 \mathrm{~mm}$. This process yielded subject-level $t$-maps for each condition, which were used for all subsequent analyses.

\section{Representational Similarity Analysis}

Neural representational dissimilarity matrices (RDMs) were calculated per subject and $\mathrm{ROI}$ by extracting voxel-wise $t$-values for each condition, and calculating the Euclidean distance between each pair of conditions using the CoSMoMVPA toolbox for MATLAB (Oosterhof et al., 2016). Neural RDMs were tested against three conceptual models of hypothesized relationships between conditions, which were formalized as binary RDMs wherein $0=$ similar and $1=$ dissimilar. Model 1 , or the "interaction elicits mentalizing" model, states that Character Mental and both Peer conditions are similar (i.e., have 0's) with each other and dissimilar (i.e., have 1's) from Character Non-mental. Model 2, or the "context-specific" model, states that both Peer conditions are similar to each other and both Character conditions are similar to each other, but Peer and Character conditions are dissimilar from each other. Model 3, or the "mentalizing" model, states that both Mental conditions are similar to each other and both Non-mental conditions are similar to each other, but Mental and Non-mental conditions are dissimilar from each 
other (Figure 2). Model fit was estimated by calculating Kendall's Tau-a rank correlation coefficient between the off-diagonal elements of the neural and model RDMs using the RSA toolbox for MATLAB (42). For each ROI, model fit was calculated between each participant's neural RDM and the three model RDMs, and the resulting estimates of model fit were analyzed using Wilcoxon signed rank tests. Specifically, the estimates of fit for each model were tested against zero using a one-sample Wilcoxon signed rank test to evaluate significant model fit, and estimates of fit were compared against each other using paired-sample Wilcoxon signed rank tests.

Mentalizing and Social Interaction Conjunction ROls. To evaluate if overlapping brain activations between social interaction and mentalizing are indicative of similar underlying representations, we conducted RSA in each of the four conjunction ROIs reported by Alkire and colleagues (11; Figure 5/Table 2, page 10), which included: 1 ) left ATL cluster extending into the insula and lateral OFC; 2) right ATL; 3) right posterior STS; 4) right IFG. First, we estimated model fit in the full sample of 72 participants via one-sample Wilcoxon signed rank tests greater than zero, and assessed significance using false discovery rate (FDR) adjusted $p$-values $<.05$ (43) for all 12 tests (i.e., 3 models x 4 ROls). Comparisons between models were conducted for each ROI using paired-sample Wilcoxon signed rank test between each pair of models using the full sample of 72 participants, and assessed using FDR adjusted $p$-values $<.05$ for the three comparisons (i.e., 3 pairwise comparisons between models for each ROI). We then calculated fit to each of our models using data from the original 28 participants included in 11 before performing the same procedure using previously unanalyzed data 
from the 44 new participants as means to assess the reliability of our findings. If the general pattern of results of the original sample is replicated by our new sample, it provides added support for the reliability of our findings.

Independent Social Interaction ROls. The next set of theory-driven analyses utilized an independent set of ROls obtained through NeuroSynth (35) to examine the similarity structures in brain regions associated with social interaction. To this end, we first binarized (threshold=0.5) and combined (addition) the meta-analytic maps from NeuroSynth association tests of the search terms "social interaction" and "social interactions" using AFNI's 3dcalc. The resulting map was thresholded to a minimum cluster size of 50 voxels (nearest neighbor=3) to retain ROls of theoretical importance while eliminating numerous small clusters. This resulted in a set of 13 ROIs: dorsomedial prefrontal cortex (dmPFC), ventromedial PFC (vmPFC), perigenual anterior cingulate cortex (pgACC), subgenual ACC ( $\mathrm{sgACC})$, bilateral temporoparietal junction (TPJ), bilateral ATL, bilateral cerebellum, left caudate, right inferior temporal gyrus (ITG), and right ventrolateral PFC (vIPFC). Within each ROI, we estimated model fit in the full sample of 72 participants using one-sample Wilcoxon signed rank tests greater than zero, and assessed significance using false discovery rate (FDR) adjusted $p$-values $<.05$ (43) for all 39 tests (i.e., 3 models $\times 13$ ROls).

Age-related effects. To examine age-related differences, correlations between model fit and age were calculated using the independent social interaction ROls rather than 
the conjunction ROls reported by Alkire and colleagues (11) to avoid the inherent circularity of using ROls obtained from our sample (44).

Searchlight. Exploratory searchlight analyses were performed using CoSMoMVPA (45) for each of the three models to reveal model fits in brain regions that were not previously considered. This approach involves extracting local patterns of brain activity throughout each participant's brain. A sphere of approximately 100 voxels was drawn around each gray matter voxel, and the voxel-wise $t$-values are extracted for each condition. A neural RDM was created by calculating the Euclidean distance between each pair of conditions, and model fit between the neural and model RDMs was estimated using CoSMoMVPA's implementation of Kendall's tau. After performing a Fisher $z$ transformation, the $z$-value was assigned to the center voxel, yielding whole-brain maps of model fit estimates throughout the brain. One-sample $t$-tests were calculated on these subject-level maps for each model using AFNl's 3dttest++ to determine significant model fits across our sample. For all group-level analyses, the cluster correction value was calculated automatically by using the -Clustsim option in AFNl's 3dttest++, which sends the input volumes directly to the 3dClustSim program after simulating the noise volumes by randomizing and permuting the input datasets. This model-free approach is more accurate at controlling the false positive rate compared to simulating noise using a mixed-model estimation of the autocorrelation function. This yielded a voxel-wise threshold of $p<.001$ and cluster extent of $k=117$ to achieve a whole-brain familywise error rate of $\alpha=0.05$ when nearest neighbor cluster 
definitions are set to 3 (i.e., voxel faces, edges, and corners touching count as part of a contiguous cluster).

Social Brain ROls. A final set of model-free analyses were conducted to evaluate if other, non-hypothesized relations between task conditions exist in the social brain, and to examine the organization of these regions based on pattern similarity structure. To this end, we started with a set of 36 publicly available ROls derived from an extensive set of meta-analytic, data-driven analyses of 3,972 social neuroscience studies using fMRI and/or PET imaging (36). The ROIs were resampled to the resolution of our functional data, and inflated within a gray matter mask informed by white matter and CSF skeleton to maintain a comparable amount of brain coverage and attention to anatomical contours. The resulting ROls had a uniform volume of 120 voxels.

Analyses of these social brain ROls proceeded in 3 major steps. First, because the social brain ROls cut across many different brain systems that may not be involved in any of our task conditions, it was necessary to calculate the lower bound of the "noise ceiling," which expresses how consistent the representations are across participants. Noise ceiling calculation was conducted for each $\mathrm{ROI}$ and tested against zero (i.e., no consistency across participants) to determine their inclusion in further analyses (46). Noise ceiling was calculated by creating an average neural RDM across all but one participant, calculating Kendall's tau between this average RDM and the neural RDM from the left-out participant, and iterating this process across participants. The Kendall's tau coefficients were then tested against zero using Wilcoxon signed rank tests, and ROls with a noise ceiling significantly above chance were retained. 
Next, the resulting ROls were interrogated using exploratory factor analysis $(E F A ; 47)$ to determine the factor structure of the response patterns across the ROIs, which elucidated the number of groups that the ROls were separated into based on representational connectivity (i.e., shared similarity structures between ROIs). This was conducted by first averaging the neural RDMs across participants for each ROI, and entering the averaged Euclidean distances into an EFA using minimum residual extraction and oblimin rotation (though results from principal axis extraction and other rotation methods yielded similar results). A cut-off eigenvalue of 1 and a minimum of $80 \%$ explained variance were used to determine the factor solution. Finally, the Euclidean distances were used to calculate correlations between each pair of ROls, which were submitted to a hierarchical cluster analysis using Ward's method (48) to identify clusters of regions with similar neural similarity structures, and the number of clusters were set to the number of factors determined from the EFA. The neural RDMs for each ROI within each cluster were averaged together and qualitatively examined for a better understanding of the neural representations of each cluster of ROls. The code for all analyses are available at https://github.com/JunaidMerchant/CMNT RSA.

\section{Results}

\section{Behavioral Data Analysis}

No significant gender effects were revealed from the independent samples $t$-tests of accuracy and RT, including tests for main and simple effects (all $p$-values $>.16$ ), thus gender was not used in additional analyses of variance. There were significant negative correlations with age for every RT measure (all $p$-values $<.004$ ) indicating that participants get faster with age. Significant positive correlations with age were revealed 
for accuracy on Mental trials, $r(70)=.26, p=.03$, and Character Mental trials, $r(70)=$ $.25, p=.04$, indicating better performance on these trials with increasing age. Age was therefore subsequently used as a covariate for the accuracy and RT analyses of variance.

A 2 (Target: Peer vs. Character) x 2 (Question type: Mental vs. Non-mental) repeated measures ANCOVA with age as a covariate on accuracy scores revealed a main effect for question type, $F(1,70)=4.83, p=.03$, indicating higher accuracy for Mental questions, and an age by question type interaction, $F(1,70)=6.99, p=.01$, such that the difference in accuracy between Mental and Non-mental questions is greater with increasing age (Figure 3A). A 2 (Target: Peer vs. Character) x 2 (Question type: Mental vs. Non-mental) repeated measures ANCOVA with age as a covariate on RT scores revealed a main effect for age, $F(1,70)=14.24, p<.001$, indicating faster overall RT with age; a significant target $\mathrm{x}$ question type interaction, $F(1,70)=4.19, p=.04$, such that there is a bigger RT difference between Mental and Non-mental for the Peer condition; and a significant three-way interaction, $F(1,70)=4.78, p=.03$, such that the difference between Peer Mental vs Peer Non-mental gets bigger with age (Figure 3B). 


\section{A. Accuracy results}

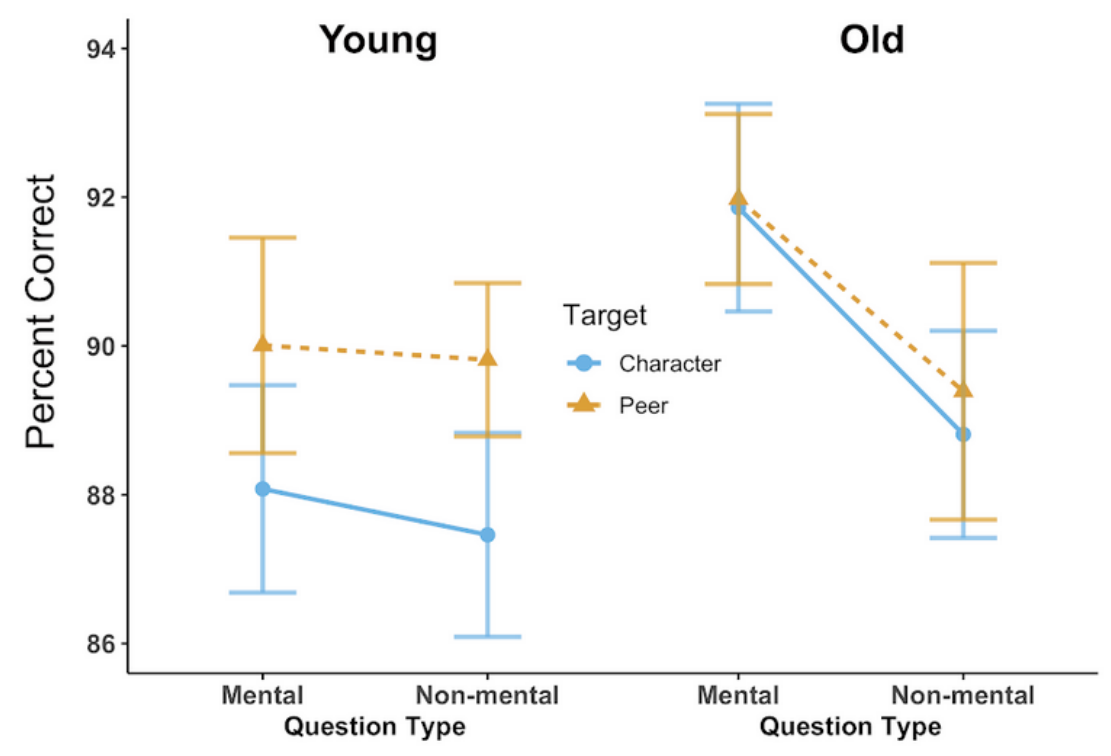

B. Reaction time results

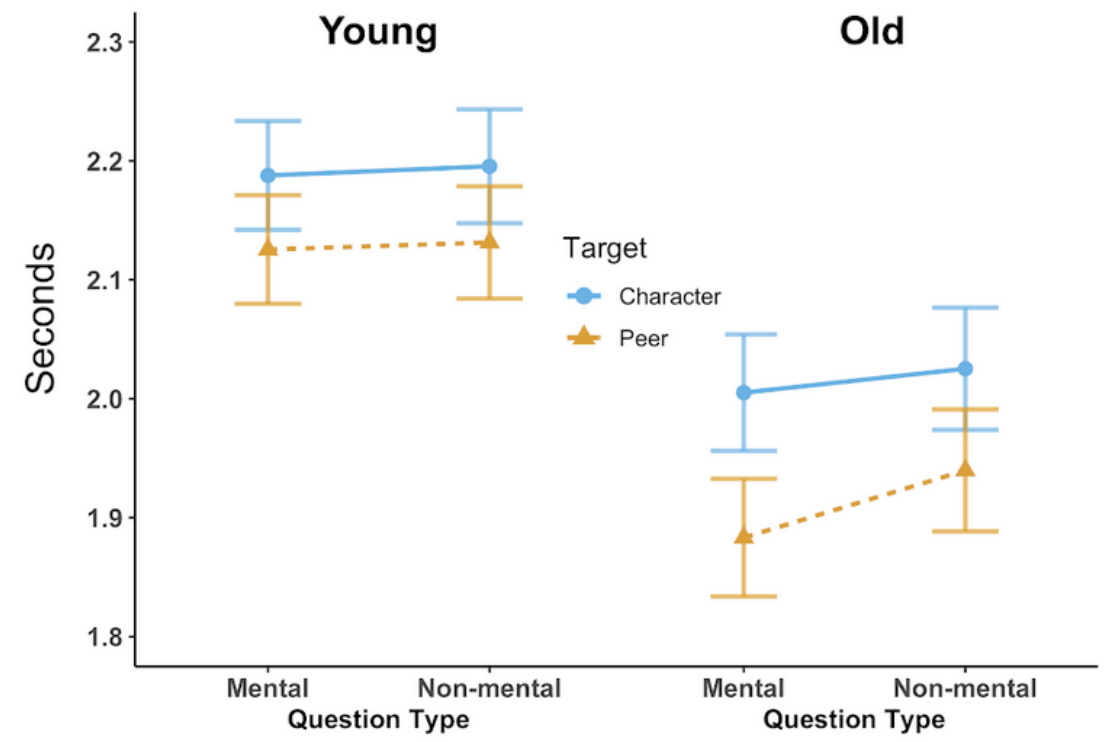

Figure 3. Visualization of the $2 \times 2$ analysis of covariance for accuracy (a) and reaction (b) 


\section{ROI - MODEL FIT ANALYSES \\ Conjunction ROls}

Left ATL. One-sample Wilcoxon signed rank test on the estimates of model fit for the full sample of 72 participants in the left ATL ROI demonstrated significant fit with Model $1, V=1714.5$, FDR adjusted $p=.045$, and Model $2, V=1061.5$, FDR adjusted $p=.045$, but model comparison tests indicated no significant differences between models (Figure 4A). Examinations of the original sample of 28 participants and the new sample of 44 participants demonstrated that this pattern of results generally replicated, such that each sub-sample exhibited a similar degree of model fit to models 1 and 2 in the left ATL; however, none of the Wilcoxon tests on the sub-samples reached the criteria of significance.

Right ATL. We found no significant fits for the right ATL to any of the models when using the full sample of 72 participants, nor did we find any significant differences between models. When examining the sub-samples of our data, we did find a significant fit to Model 2 in the original sample of 28 participants, but this did not replicate in the new sample of 44 participants (Figure 4B).

Right IFG. One-sample Wilcoxon signed rank test on the estimates of model fit for the full sample of 72 participants in the right IFG ROI revealed significant fit with Model 2, $\mathrm{V}$ $=1039$, FDR adjusted $p=.023$, and model comparison tests demonstrated significantly better fit for Model 2 than Model 3, $V=1266$, FDR adjusted $p=.003$, and marginally better fit than Model 1, V =935.5, FDR adjusted $p=.051$ (Figure 4C). Analysis of the sub-samples demonstrated that this pattern of model fits to model 2 in right IFG 
generally replicated between the original sample of 28 and the new sample of 44 , such that each sub-sample exhibited a similar degree of model fit.

Right pSTS. One-sample Wilcoxon signed rank test on the estimates of model fit for the full sample of 72 participants in the right pSTS ROI revealed significant fit with Model 1 , , $V=1780$, FDR adjusted $p=.026$, and model comparison tests demonstrated better fit for Model 1 compared to Model 3, $V=1687$, uncorrected $p=.036$ (though not after FDR adjustment, $p=.107)$. Analysis of the sub-samples demonstrated that this pattern of results (i.e., best fit with model 1 ) generally replicated between the original sample of 28 and the new sample of 44 , such that each sub-sample exhibited a similar degree of model fit (Figure 4D). 

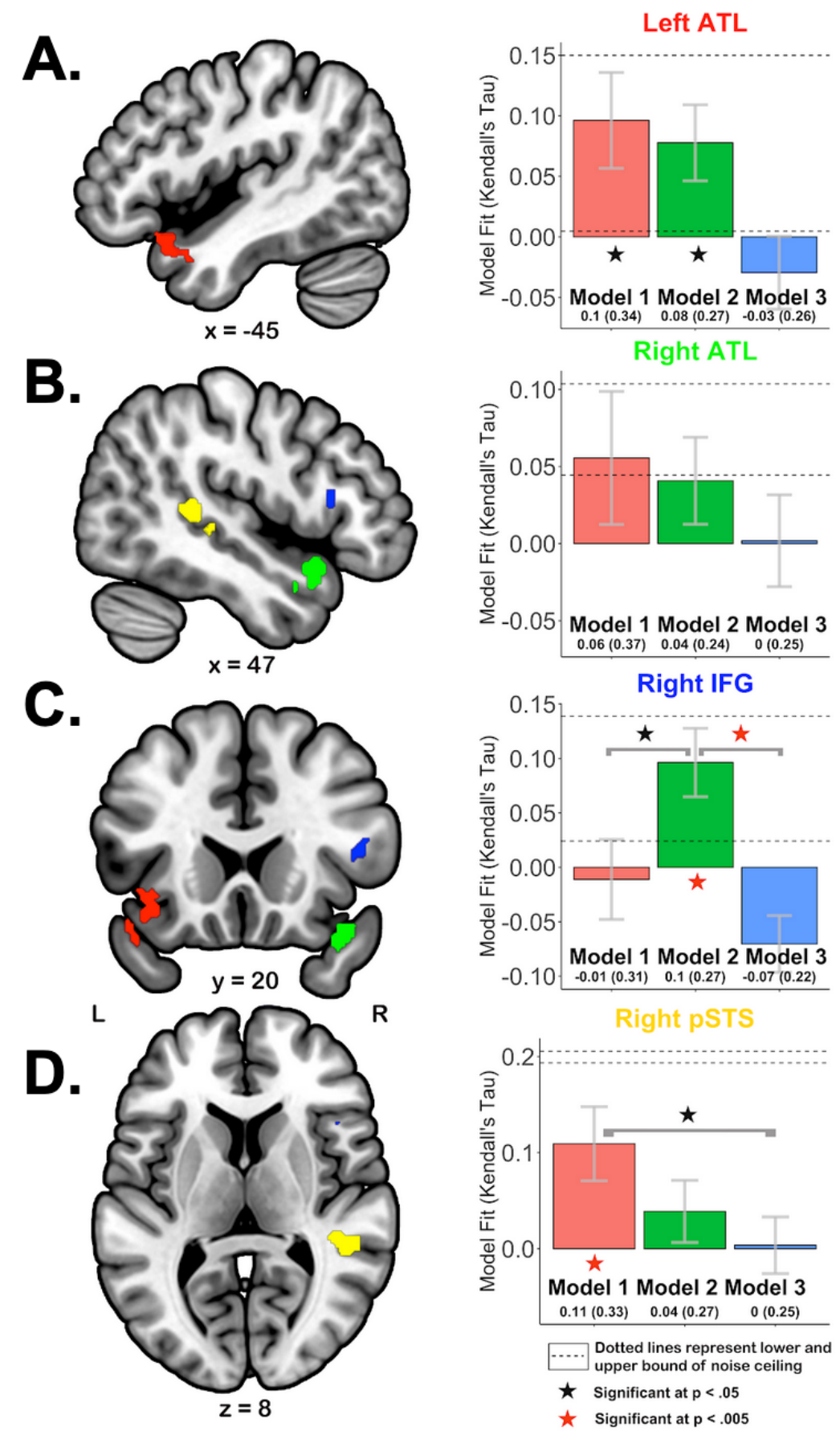

Figure 4. Conjunction ROls model fit for the left anterior temporal lobe (a), right anterior temporal lobe (b), right inferior frontal gyrus (c), and right posterior superior temporal gyrus. 


\section{Neurosynth Social Interaction ROls}

Across all 13 of the social interaction ROls, 4 showed significant fit with Model 1: left ATL, $V=1744$, FDR adjusted $p=.033$; left TPJ, $V=1782.5$, FDR adjusted $p=.022$; right ATL, $V=1821$, FDR adjusted $p=.016$; and marginally in the right TPJ, $V=1661$, uncorrected $p=.025$ (FDR adjusted $p=.096$ ). Seven ROls showed significant fit with Model 2: dmPFC, $V=1283.5$, FDR adjusted $p=.020$; left caudate, $V=1025.5$, FDR adjusted $p=.033$; left cerebellum, $V=1331.5$, FDR adjusted $p=.002$; left TPJ, $V=$ 1112, FDR adjusted $p=.003 ; \operatorname{pgACC}, V=1021.5$, FDR adjusted $p=.011 ;$ right TPJ, $V$ $=1209.5$, FDR adjusted $p=.002$; and marginally in the right vIPFC, $V=1032$, uncorrected $p=.027$ (FDR adjusted $p=.096$ ). The bilateral TPJ clusters showed fits to Model 1 and 2 and model comparisons revealed no significant differences between the two models. The only ROI showing significant differences in fit between models was the left cerebellum, which showed a significantly stronger fit for Model 2 than Model $1, W=$ 1735.5, FDR adjusted $p=.027$, and Model $3, W=1568$, FDR adjusted $p=.003$, and stronger fit for Model 1 than Model 3, $W=1705.5$, FDR adjusted $p=.028$ (Figure 5).

\section{Age-related differences in model fits.}

Of the neurosynth social interaction ROls showing significant model fits, two showed significant negative correlations with age: the left caudate showed a significant negative correlation between age and Model 2 fit, $r_{s}(70)=-.323, p=.006$, and the right vIPFC showed a significant negative correlation between age and Model 2 fit, $r_{s}(70)=-.298, p$ $=.011$. Follow up, exploratory linear regression analyses for each ROI were conducted 
to determine which, if any, of the distances between conditions drove these effects. Age was used as the dependent variable and the Euclidean distance between each pair of conditions were used as independent variables. The regression model for the right VIPFC found a significant relationship with age, $F(6,65)=2.57, p=.027, R^{2}=.19$, and partial model coefficients indicate that this effect was driven by a negative relationship with the distance between Character Mental and Peer Non-mental, $B=-.85, \mathrm{SE}=.3$, $95 \% \mathrm{Cl}[-1.45,-.25], \mathrm{t}=-2.84, \mathrm{p}=0.006$ and a positive relationship with the distance between Peer Mental and Peer Non-mental, B $=.86$, SE $=.27,95 \% \mathrm{Cl}[.33,1.39], \mathrm{t}=$ $3.24, p=0.002$. Similarly, the regression model for the left caudate found a significant relationship with age, $F(6,65)=2.25, p=.049, R^{2}=.17$, and partial model coefficients indicate that this effect was driven by a positive relationship with the distance between Peer Mental and Peer Non-mental, B $=.99, \mathrm{SE}=.32,95 \% \mathrm{Cl}[.36,1.62], \mathrm{t}=3.14, \mathrm{p}=$ 0.003. Together, these results suggest that the response patterns associated with the Peer Mental and Peer Non-mental conditions might become more distinct with age (Figure 5). 


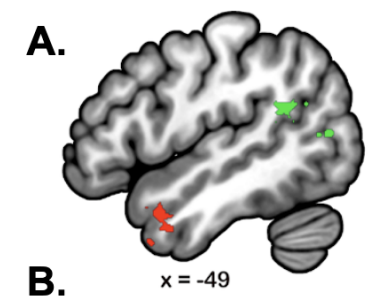

B.

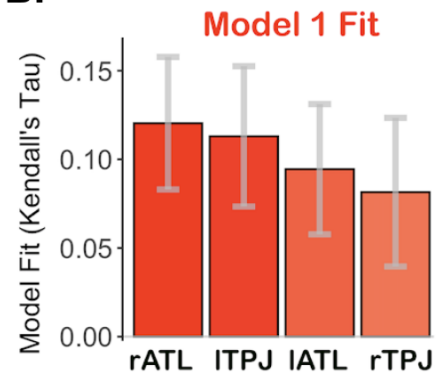

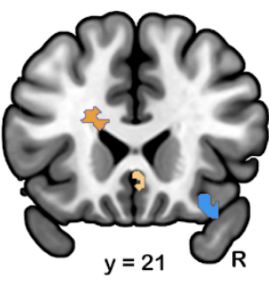

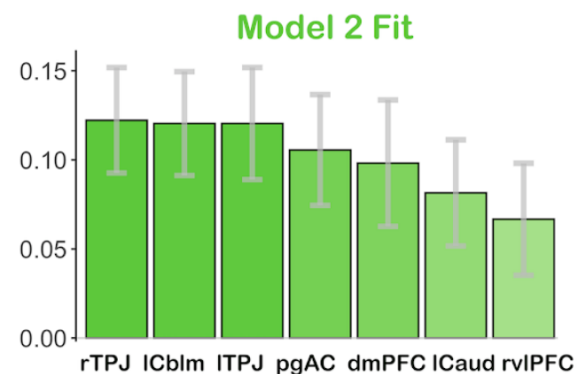

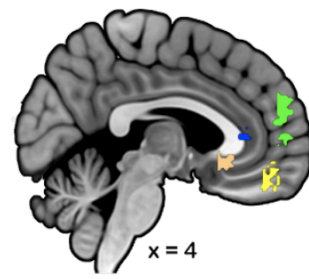

Model 2 Fit

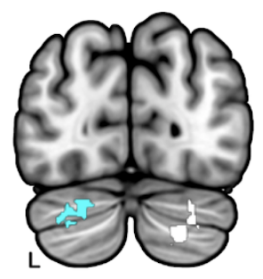

C. Age by Model 2 Fit in Left Caudate

NeuroSynth ROIs: Dorsomedial Prefrontal Cortex (dmPFC), Left Anterior Temporal Lobe (IATL), Left Caudate (ICaud), Left Cerebellum (I $\mathrm{Crb} / \mathrm{m})$, Left Temporoparietal Junction (ITPJ), Perigenual Anterior Cingulate (pgACC), Right Anterior Temporal Lobe (rATL), Right Cerebellum ( $\mathrm{rCrb} / \mathrm{m})$, Right Inferior Temporal Gyrus ( $\mathrm{rlTG}$ ), Right Temporoparietal Junction (rTPJ), Right Ventrolateral Prefrontal Cortex (rvIPFC), Subgenual Anterior Cinguate ( $\mathrm{g} A \mathrm{ACC})$, Ventromedial Prefrontal Cortex (vmPFC)

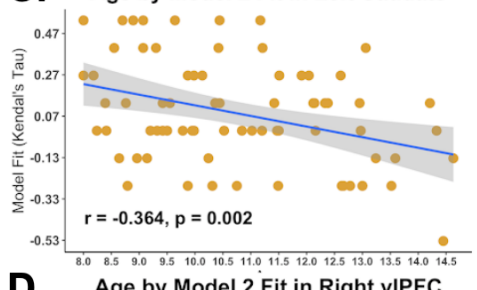

D. Age by Model 2 Fit in Right vIPFC

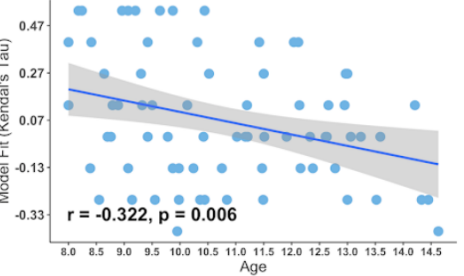

Figure 5. Social interaction ROIs obtained from Neurosynth (a), and their fits to Models 1 and 2 (b). Correlations between age and model fit for the left caudate (c) and the right ventrolateral prefrontal cortex (d).

\section{WHOLE-BRAIN SEARCH FOR MODEL FIT - Searchlight Analysis}

Searchlight analysis for Model 1 yielded significant model fit in a 128-voxel cluster in the left ATL, supporting the findings from the ROI analyses. Exploratory examination of the group-level models at a reduced, uncorrected threshold $p<.005, \mathrm{k}=150$ revealed additional clusters in the right ATL and right pSTS, though these findings should be interpreted with caution given the uncorrected threshold. Searchlight analysis for Model 2 revealed 12 clusters showing significant model fit, including a cluster encompassing much of the visual cortex, a cluster going from dorsal to ventromedial PFC, left 
cerebellum, bilateral TPJ, left pSTS, left IFG, left precentral gyrus, right Al, supplementary motor area, and bilateral dorsal frontal pole. Searchlight analysis for Model 3 fit yielded no significant clusters, even at exploratory, uncorrected thresholds. Group-level comparison between Models 1 and 2 revealed a large cluster in the visual cortex showing a significantly greater fit for Model 2 (Figure 6, Table 2).

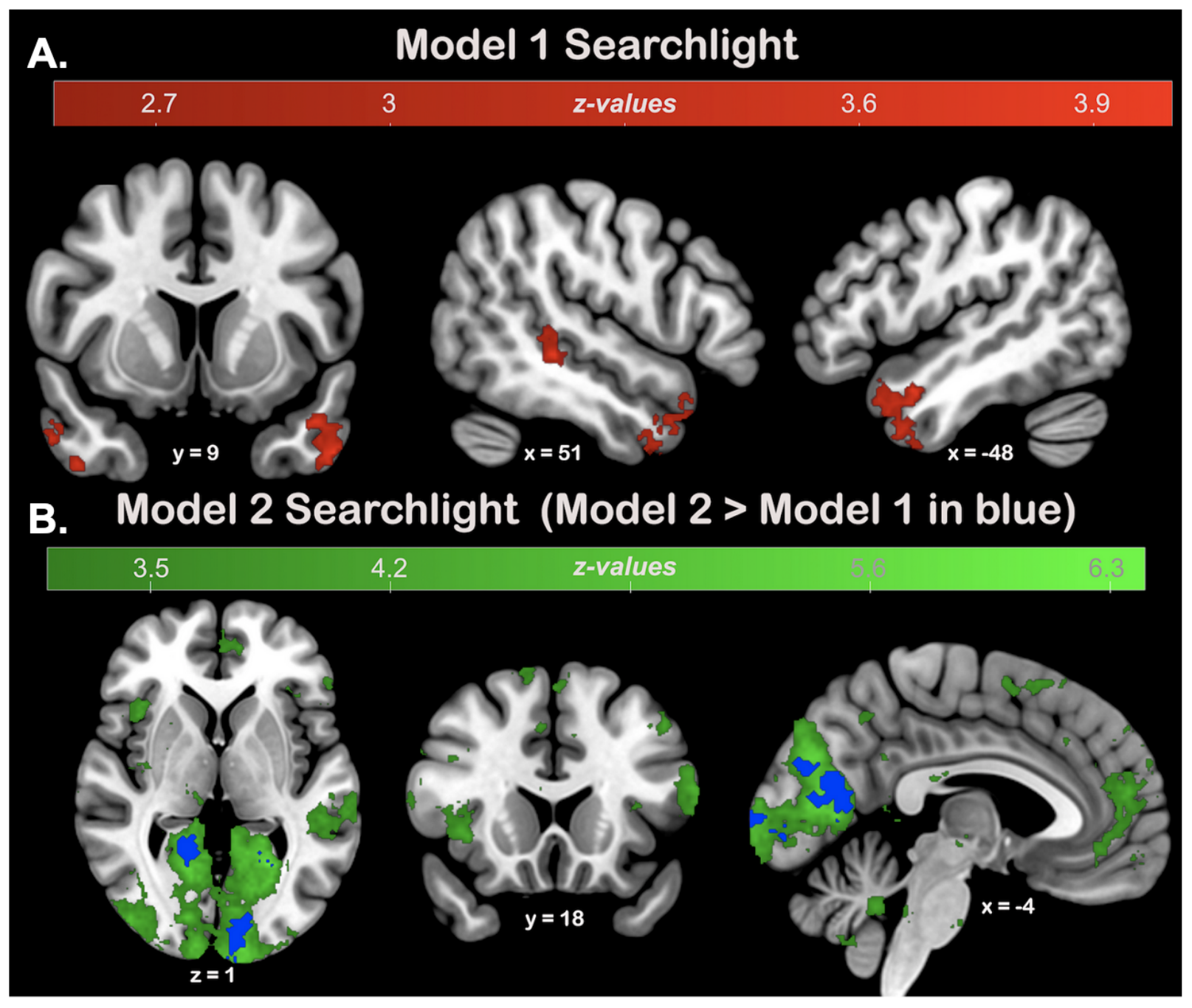

Figure 6. Searchlight results for Model 1 (a) and Model 2 (b) 


\begin{tabular}{|c|c|c|c|c|c|c|}
\hline \multicolumn{4}{|c|}{ A) Model 1 Searchlight, $p<.001, k=117$} & & & \\
\hline \multirow[b]{2}{*}{ Region } & \multirow[b]{2}{*}{ Hemisphere } & \multirow[b]{2}{*}{ Cluster Size (voxels) } & \multirow[b]{2}{*}{ Peak z } & \multicolumn{3}{|c|}{ MNI Coordinates (center of mass) } \\
\hline & & & & $\mathbf{x}$ & y & $\mathbf{z}$ \\
\hline \multicolumn{7}{|l|}{ Regions with significant model fit } \\
\hline Anterior Temporal Lobe & L & 128 & & -49 & 6 & 31 \\
\hline posterior Superior Temporal Sulcus* & $\mathrm{R}$ & 209 & & 47 & 34 & 0 \\
\hline Anterior Temporal Lobe* & $\mathrm{R}$ & 157 & & 53 & 8 & -33 \\
\hline \multicolumn{7}{|c|}{ *Not significant at cluster corrected threshold. Only for exploration: $\mathrm{p}<.005, \mathrm{k}=150$} \\
\hline \multicolumn{7}{|c|}{ B) A) Model 2 Searchlight, $p<.001, k=117$} \\
\hline \multirow[b]{2}{*}{ Region } & \multirow[b]{2}{*}{ Hemisphere } & \multirow[b]{2}{*}{ Cluster Size (voxels) } & \multirow[b]{2}{*}{ Peak $t$} & \multicolumn{3}{|c|}{ MNI Coordinates (center of mass) } \\
\hline & & & & $\mathbf{x}$ & y & $\mathbf{z}$ \\
\hline \multicolumn{7}{|l|}{ Regions with significant model fit } \\
\hline & $\mathrm{R}$ & 11803 & & 3.1 & -75.5 & 6.3 \\
\hline & $\mathrm{R}$ & 928 & & 4.7 & 41.6 & 22.5 \\
\hline & L & 433 & & -35.2 & -67.8 & -50.7 \\
\hline & L & 403 & & -56.1 & -51.8 & 26.1 \\
\hline & L & 381 & & -56.7 & -34.5 & 0.7 \\
\hline & L & 372 & & -51.5 & 21.4 & 11 \\
\hline & $R$ & 353 & & 56.7 & -47.5 & 26.7 \\
\hline & $\mathrm{R}$ & 213 & & 40.3 & 20.3 & 0.5 \\
\hline & L & 195 & & -43.4 & 14.4 & 48 \\
\hline & L & 191 & & -1.2 & 13.2 & 61.9 \\
\hline & $\mathrm{L}$ & 150 & & -17.7 & 45 & 38.9 \\
\hline & $\mathrm{R}$ & 142 & & 25.8 & 51 & 30 \\
\hline
\end{tabular}

Table 2. Clusters showing significant fit to Model 1 (a) and Model 2 (b).

\section{Model free analyses within the Social Brain}

Analyses to examine the similarity structure of the social brain ROIs proceeded in 3 main steps: 1) ROI selection based on significantly greater than zero noise ceiling; 2) exploratory factor analysis to determine the number of factors underlying the similarity structures of the selected ROls; 3) hierarchical clustering and qualitative examination of the similarity structures of the ROls clustered together.

Noise ceiling calculation revealed that 16 of the 36 ROls were significantly above chance, suggesting that these ROls contain consistent representations across our sample. This included bilateral ATL, bilateral pSTS, bilateral inferior parietal lobule (IPL), 
bilateral cerebellum, frontal pole, dmPFC, precuneus, left AI, left hippocampus, left middle temporal gyrus (MTG), posterior mid-cingulate (pMC), and right amygdala. Thus, these 16 were included in the next step of exploratory factor analysis (Figure 7A). Exploratory factor analysis of these 16 ROls yielded a 3-factor solution that explained $88.5 \%$ of the variance in Euclidean distances. Hierarchical clustering using Ward's method revealed three clusters (Figure 7B). Qualitative examination of the averaged Euclidean distances of each cluster of ROls revealed that Cluster 1 (bilateral ATL and right pSTS) resembled Model 1, in that PM, CM, \& PNM were similar to each other, and different from CNM. Cluster 2 (left Al, left hippocampus, left pSTS, left MTG, dmPFC, right amygdala, and right IPL) showed the reverse pattern such that, while Peer and Character conditions were dissimilar from each other, the Character conditions were much more similar to each other than the Peer conditions. The latter two Clusters suggest that, while these ROls might show a significant fit to Model 2, their similarity structures are distinct. Cluster 3 (bilateral cerebellum, left IPL, precuneus, frontal pole, and $\mathrm{pMC}$ ) showed a resemblance to Model 2 in that Peer and Character conditions were dissimilar; however, the Peer conditions were much more similar to each other than the Character conditions (Figure 7C). 
A. Regions of interest

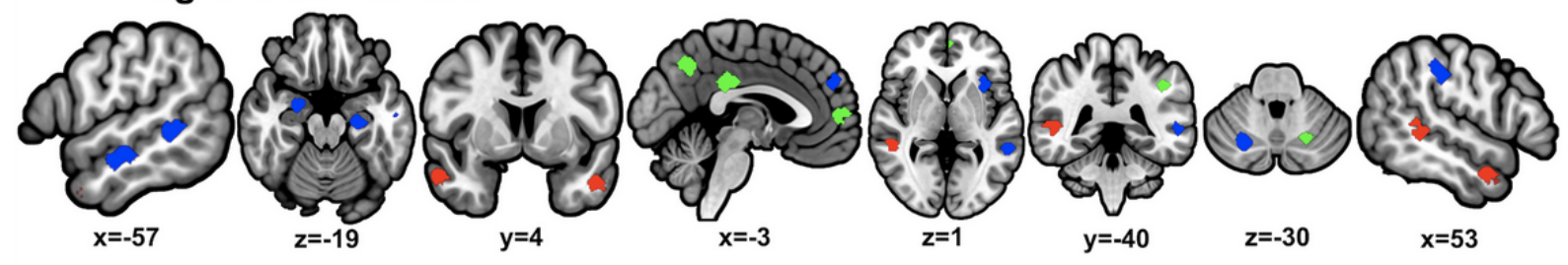

B. Factor analysis and clustering

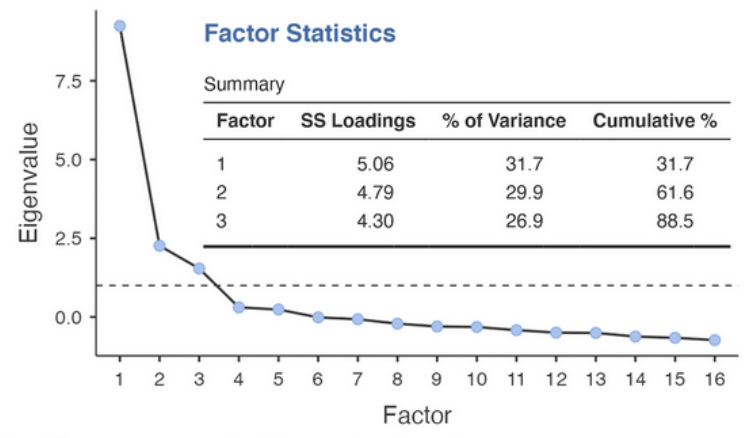

C. Representational clusters
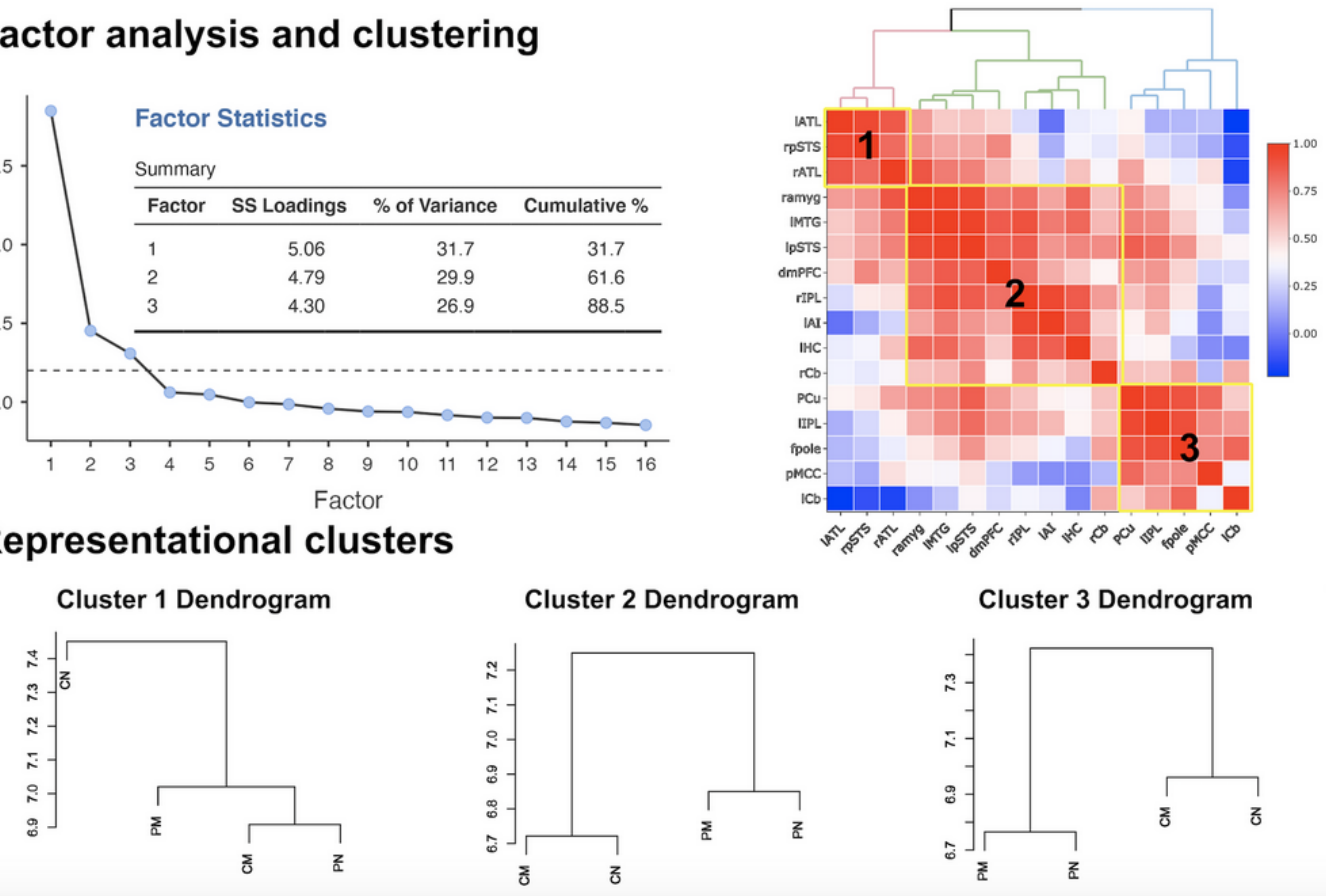

Figure 7. Social brain regions of interest used in representational connectivity analysis (a), and the results from the exploratory factor analysis and clustering (b). Dendrograms from the averaged dissimilarities for the ROls comprising each of the 3 clusters (c).

\section{Discussion}

This study evaluated the relationship between social interaction and mentalizing across a series of analyses that examined the pattern of brain activity elicited by each. Through quantifying the similarity of voxel-wise BOLD response across conditions, we assessed how similarly or distinctly different brain regions responded during social 
interaction and mentalizing, and tested conceptual models of how the two relate. We found that the ATL and pSTS exhibited a similar pattern of response during social interactions with a peer and when mentalizing about an abstract character, suggesting that mentalizing may be a constituent process for social interactions. We also found numerous brain regions associated with social cognition that demonstrated distinct response patterns between the interaction partner and abstract story character, suggesting context-specific neural representations (i.e., similar responses within the peer conditions that differ from the character). Moreover, in some of these regions, there were dissimilar response patterns when making mental and non-mental inferences about one target (either peer or character), but not the other. Surprisingly, however, we found no brain regions that exhibited separable neural representations for mentalizing and non-mental state reasoning that cut across targets.

This is the first study to evaluate the assumption that mentalizing is automatically engaged during social interactions using RSA. We found consistent evidence that offline mentalizing and live peer interactions elicit similar patterns of brain activity in the ATL and right pSTS, but a dissimilar pattern of activation when making non-mental state inferences about an abstract character (i.e., the "interaction elicits mentalizing" model or Model 1). Our findings align with traditional, offline studies of mentalizing that have demonstrated differences in brain activity in these regions when making mental versus non-mental inferences about abstract others (49-51), but add to the literature by demonstrating that patterns similar to mentalizing are elicited during social interactions that do not explicitly require mental state reasoning. This set of results provides additional evidence that mentalizing is spontaneously engaged in social interactive 
contexts, and demonstrates a neuro-representational link between separate lines of research that have implicated the ATL and pSTS during offline mentalizing and engagement with a social partner in real time $(7,9,11)$. Moreover, our findings suggest that these temporal regions may function as the convergence point between online and offline social cognitive processes. That is, social interactions involve the dynamic interplay between social knowledge that is available offline, such as an understanding of mental state concepts, and the online demands of reciprocal social exchange. This interpretation also fits with recent reviews describing how we get to know others, which involves acquiring and updating person-specific knowledge through online engagements with them, and implicates the ATL and pSTS in this process $(52,53)$.

Consistent with the idea that the ATL supports the integration of online and offline social processes, different lines of research have demonstrated the convergence of multimodal, social information processing streams in the anterior portions of the temporal lobe. Ventral areas of the ATL are involved in the encoding and retrieval of person-specific knowledge (e.g., name and biographical information; 16,21,54,55), while more dorsal, anterior portions of the ATL are considered a semantic hub for abstract social knowledge $(14,18,56-60)$. Our findings span the middle ground between the aforementioned lines of research, such that our findings suggest an integration of person-specific and abstract social knowledge representations. That is, the functional accounts of the dorsal ATL can explain pattern similarities between the two mentalizing conditions (i.e., peer mental and character mental) that involve the processing of social semantic information (i.e., mental state concepts), while the person-specific representations in the ventral ATL suggests pattern similarity for the two peer conditions 
(i.e., peer mental and peer non-mental). Indeed, research suggests that the interplay between ventral and dorsal ATL contributes to the successful encoding and retrieval of abstract social information associated with a person's identity $(19,61,62)$. Furthermore, we found our most consistent results in the left ATL, which can be explained by research demonstrating left-lateralized sensitivity for name-based identification of individuals $(18,63-65)$ since our experimental paradigm used visually presented names to specify target condition.

An alternative account for our ATL findings is that both mental state information and social information about a social partner (but not character) are processed as social semantic information in the ATL. Indeed, the ATL regions we examined were spatially closer to the dorsal, anterior regions associated with processing social semantic knowledge than the ventral regions associated with person-specific knowledge representation (though ours were more polar). Moreover, research has demonstrated that non-social concepts can engage the dorsal ATL when they are imbued with social meaning $(18,57)$. Thus, the pattern similarity of the Peer Non-mental condition with the two Mental conditions may reflect that non-mental attributes are spontaneously imbued with abstract social meaning in social-interactive contexts. This spontaneous attribution may not be present in non-interactive contexts. Furthermore, prior research on person-specific knowledge representation utilized experimental paradigms wherein participants were given explicit instructions to learn about abstract characters $(21,62)$, suggesting an active process that may not naturally emerge in social interactive contexts. Future research could probe whether person-specific representations 
crystallize within interactive contexts by examining changes in response patterns throughout the course of an experiment.

The pSTS, on the other hand, has traditionally been associated with the visual perception of biological motion (66-69) and social interactions (70-72), and is considered part of a third visual pathway that is specialized for social information (73). Beyond its association with visual social cognition, the pSTS has been implicated in the haptic exploration of face stimuli by blind individuals (74), multimodal sensory integration $(15,19)$, and social interpretations of stimuli that are not inherently social $(71,75)$. Therefore, the pSTS can be thought of as a perceptual analogue to the social semantic integration occurring in the ATL in that it gives social meaning to perceived behaviors, and its role in social interaction is associated with inferring intentionality, including communicative intent, from another's actions. Indeed, the placement of the pSTS at the intersection of the mentalizing, mirror neuron, social perceptual, and communicative systems (76-78) provides support for this interpretation. Notably, however, despite the fact that our stimuli were presented visually, they were social only in their symbolic content. Even though this is seemingly at odds with the role of the pSTS in social perceptual processes as outlined above, studies using similarly abstract tasks have also implicated the pSTS $(49-51,77)$. Together, the combination of social cognitive functions associated with the pSTS, namely its involvement in inferring intentionality and the perception of social interaction, supports the interpretation that it serves as a link between mentalizing and social interaction.

Many of the brain regions we examined demonstrated a significant fit to Model 2, which assumes dissimilar representations for the interaction partner and an abstract 
character, but does not distinguish between mental and non-mental state reasoning for either target. On the surface, Model 2 aligns with studies demonstrating representations of person-specific knowledge that have implicated the ATL and pSTS, but our results demonstrated inconsistent fit with Model 2 in these regions (i.e., only in the conjunction ROIs). Many brain areas beyond the canonical mentalizing regions exhibited Model 2 fit, including nodes of the fronto-parietal network (i.e., IFG, dIPFC, and IPL), reward and affective systems (i.e., caudate and amygdala), as well as a large swath of the visual cortex. Distinct response patterns for peer and character conditions that span across multiple brain networks may highlight the distinction between real time engagement with a social partner, and offline assessments of an unknown character. That is, while Model 2 fit in the visual cortex reflects the sensitivity of RSA to lower-level visual dissimilarities between the stimuli used in peer and character conditions (i.e., differences in color and shape of the hints), Model 2 fits in higher-order social cognitive brain regions highlight substantial differences between task conditions in their social cognitive demand. Consistent with the premise of second-person neuroscience approaches that assume fundamental differences in social cognitive processes within live, interactive contexts, engaging in real-time social interaction compared to thinking about an abstract character likely heightens attention, motivation, and social cognitive demands, which may contribute to pattern dissimilarity between target conditions $(6,7)$.

Additional support for the assumption of second-person neuroscience (i.e., that social cognition is fundamentally different during social interaction) comes from our model-free analyses that revealed two different variations of what we had collapsed together as Model 2: one variant in which the representations for Character Mental and 
Character Non-mental are distinct while the two Peer conditions are relatively similar, and a second variant showing the opposite pattern that Peer Mental and Peer Non-mental are distinct while the two Character conditions are similar. The cluster of ROls comprising the first variant (i.e., distinguishing Mental and Non-mental conditions for the Character) includes cortical midline structures typically associated with offline social cognitive processes (i.e., posterior cingulate, precuneus, and frontal pole), as well as bilateral cerebellum and the left IPL/SMG. This similarity structure could be interpreted as being in line with traditional studies of self/other appraisals that have participants make offline evaluations about others, and have uncovered differences in many of the brain regions mentioned above $(29,79-81)$. A larger number of ROls comprise the second variant that distinguishes the two peer conditions, which span a wider range of associated socio-emotional functions, including mentalizing, memory, affective, social perceptual, and empathic processes. Thus, these data suggest that distinctions between Mental and Non-mental (social) information differ during online (Peer) compared to offline (Character) contexts. While it makes intuitive sense that many more neurocognitive systems may be involved in representing distinct aspects of a real-life social interaction partner compared to what is involved in thinking about abstract characters, additional research is needed to validate this interpretation, as these model-free analyses were exploratory.

It is noteworthy that we did not find any significant fits to Model 3 , despite the vast literature on mentalizing that indicates mental state representations are not person-specific. For instance, a recent study demonstrated stable mental-state representations in the TPJ, ATL, and dIPFC that cut across targets over three different 
studies (30). There are a few factors that can explain this lack of finding. As previously mentioned, the majority of research on mentalizing has relied on non-interactive experimental paradigms, including the above mentioned study, so it is possible that mentalizing about a social partner in real time is different from mentalizing about a character. Furthermore, as indicated by the model-free representational connectivity analysis, it seems that the dissimilarity in response pattern between target conditions (i.e., Model 2) may have hampered our ability to see more subtle differences in response patterns associated with mental and non-mental reasoning. Moreover, our experimental paradigm, which included relatively easy, one-sentence hints, does not match previous mentalizing research that used longer, paragraph-long verbal vignettes to induce mental state reasoning $(49-51,82)$. Finally, the questions comprising the mental condition were deliberately varied to cover a range of mental state categories, including wants, likes, knowledge, beliefs, and emotions, while the Non-mental items asked about activities, physical characteristics, abilities, situations, and possessions. Thus, it is possible that this variety led to non-representative response patterns when averaged across items for each condition. Future work could probe this possibility through dimensional analysis of the neural response patterns that cut across items in each condition.

Finally, the current study provides some insight into the development of social cognitive processes. Our behavioral findings are consistent with previous work suggesting that as individuals transition from middle childhood to adolescence, they become better at making mental state inferences (31), which was reflected in our analysis of accuracy scores that demonstrated a significant age by question type 
interaction (i.e., larger accuracy score differences between Mental and Non-mental questions with age). Moreover, while it is not surprising that reaction time quickens with age, the significant three-way interaction between age, question type, and target uncovered by our RT ANCOVA demonstrates that participants become particularly faster for making Mental versus Non-mental state inferences about their interaction partner. This pattern of greater difference between Mental and Non-mental conditions for the peer specifically is also reflected in the decreasing Model 2 fit with age for the right VIPFC and left caudate. This age-related finding was driven by increasing dissimilarity between the response patterns associated with Peer Mental and Peer Non-mental conditions. Because the vIPFC and caudate are associated with reward and valuation $(83,84)$, this set of findings may reflect the ever increasing importance of peers, and particularly the need to understand their perspectives, during this age range. In line with this interpretation, research has demonstrated heightened sensitivity of the reward system to social contexts during the transition into adolescence (85-87), and a related increase in the tendency to automatically integrate peer perspectives into self-evaluations $(79,88,89)$. Thus, the current findings add to our understanding of the development of social cognition by demonstrating that improvements in mentalizing capacity during the transition to adolescence are particularly salient in naturalistic, social interactive contexts.

Despite the contributions of the current findings to our understanding of the neuroscience of social interaction, this study is not without limitations. While neural pattern similarity in the temporal lobe structures provides additional support for the assumption that social interactions may spontaneously elicit mentalizing, similar 
activation patterns are not a direct measure of common underlying neurocognitive function. Thus, future work will need to establish more precise neurocognitive measures of the mentalizing process, and assess if they are engaged during interactions that do not explicitly require mental state reasoning. Nonetheless, our findings highlight the utility of pattern-based approaches. For example, we found that the pattern of activity in the right IFG indicates context-specific representations (i.e., Model 2) that disregard the Mental/Non-mental distinction, despite previous reports of overlapping activations in this region for mentalizing and social interaction (Alkire et al., 2018).

Together, the current study advances the field of developmental social cognitive neuroscience. Our findings demonstrate the importance of incorporating realistic, social interactive contexts for a better understanding of the neural substrates subserving everyday social cognition, and highlight the utility of pattern similarity based analyses for examining the relationship between related social cognitive processes. Through this set of approaches, we provided additional evidence for the automatic engagement of mentalizing during social interactions, and spotlight the functional contributions of the ATL and pSTS toward integrating offline social cognition with online social interactions. Future work could build on these findings by establishing more precise neural and behavioral measures of mentalizing and related social cognitive processes to better verify process-level similarities and differences between them. By elucidating the links and dissociations between the various cognitive mechanisms underlying social interactions, we will gain not only a better understanding of the social behaviors that shape our world, but also a more complete cognitive map of how different neurocognitive functions relate to each other. 


\section{References}

1. Premack D, Woodruff G. Does the chimpanzee have a theory of mind? Behav Brain Sci. 1978 Dec;1(4):515-26.

2. Gallagher HL, Frith CD. Functional imaging of 'theory of mind.' Trends Cogn Sci. 2003 Feb 1;7(2):77-83.

3. Mar RA. The Neural Bases of Social Cognition and Story Comprehension. Annu Rev Psychol. 2011;62(1):103-34.

4. Molenberghs $P$, Johnson $H$, Henry JD, Mattingley JB. Understanding the minds of others: A neuroimaging meta-analysis. Neurosci Biobehav Rev. 2016 Jun 1;65:276-91.

5. Schurz M, Radua J, Aichhorn M, Richlan F, Perner J. Fractionating theory of mind: A meta-analysis of functional brain imaging studies. Neurosci Biobehav Rev. 2014 May 1;42:9-34.

6. Schilbach L, Timmermans B, Reddy V, Costall A, Bente G, Schlicht T, et al. Toward a second-person neuroscience. Behav Brain Sci. 2013 Aug;36(4):393-414.

7. Redcay E, Schilbach L. Using second-person neuroscience to elucidate the mechanisms of social interaction. Nat Rev Neurosci. 2019 Aug;20(8):495-505.

8. Redcay E, Dodell-Feder D, Pearrow MJ, Mavros PL, Kleiner M, Gabrieli JDE, et al. Live face-to-face interaction during fMRI: A new tool for social cognitive neuroscience. Neurolmage. 2010 May 1;50(4):1639-47.

9. Warnell KR, Sadikova E, Redcay E. Let's chat: developmental neural bases of social motivation during real-time peer interaction. Dev Sci. 2018;21(3):e12581.

10. Redcay E, Warnell KR. Chapter One - A Social-Interactive Neuroscience Approach to Understanding the Developing Brain. In: Benson JB, editor. Advances in Child Development and Behavior [Internet]. JAl; 2018 [cited 2020 May 13]. p. 1-44. Available from: http://www.sciencedirect.com/science/article/pii/S0065240717300319

11. Alkire $D$, Levitas $D$, Warnell KR, Redcay $E$. Social interaction recruits mentalizing and reward systems in middle childhood. Hum Brain Mapp. 2018 Oct;39(10):3928-42.

12. Hutzler F. Reverse inference is not a fallacy per se: Cognitive processes can be inferred from functional imaging data. Neurolmage. 2014 Jan 1;84:1061-9.

13. Poldrack RA. Can cognitive processes be inferred from neuroimaging data? Trends Cogn Sci. 2006 Feb 1;10(2):59-63.

14. Anzellotti $\mathrm{S}$. Anterior temporal lobe and the representation of knowledge about people. Proc Natl Acad Sci. 2017 Apr 18;114(16):4042-4.

15. Anzellotti S, Caramazza A. Multimodal representations of person identity individuated with fMRI. Cortex. 2017 Apr 1;89:85-97.

16. Blank $\mathrm{H}$, Kiebel SJ, Kriegstein $\mathrm{K}$ von. How the human brain exchanges information across sensory modalities to recognize other people. Hum Brain Mapp. 2015;36(1):324-39.

17. Collins JA, Koski JE, Olson IR. More Than Meets the Eye: The Merging of Perceptual and Conceptual Knowledge in the Anterior Temporal Face Area. Front Hum Neurosci [Internet]. 2016 [cited 2020 Nov 24];10. Available from: https://www.frontiersin.org/articles/10.3389/fnhum.2016.00189/full 
18. Olson IR, McCoy D, Klobusicky E, Ross LA. Social cognition and the anterior temporal lobes: a review and theoretical framework. Soc Cogn Affect Neurosci. 2013 Feb 1;8(2):123-33.

19. Perrodin C, Kayser C, Abel TJ, Logothetis NK, Petkov Cl. Who is That? Brain Networks and Mechanisms for Identifying Individuals. Trends Cogn Sci. 2015 Dec 1;19(12):783-96.

20. Simmons WK, Reddish M, Bellgowan PSF, Martin A. The Selectivity and Functional Connectivity of the Anterior Temporal Lobes. Cereb Cortex. 2010 Apr 1;20(4):813-25.

21. Wang Y, Collins JA, Koski J, Nugiel T, Metoki A, Olson IR. Dynamic neural architecture for social knowledge retrieval. Proc Natl Acad Sci. 2017 Apr 18;114(16):E3305-14.

22. Breitling C, Zaehle T, Dannhauer M, Tegelbeckers J, Flechtner H-H, Krauel K. Comparison between conventional and HD-tDCS of the right inferior frontal gyrus in children and adolescents with ADHD. Clin Neurophysiol. 2020 May 1;131(5):1146-54.

23. Drummond NM, Cressman EK, Carlsen AN. Offline continuous theta burst stimulation over right inferior frontal gyrus and pre-supplementary motor area impairs inhibition during a go/no-go task. Neuropsychologia. 2017 May 1;99:360-7.

24. Hartwigsen G, Neef NE, Camilleri JA, Margulies DS, Eickhoff SB. Functional Segregation of the Right Inferior Frontal Gyrus: Evidence From Coactivation-Based Parcellation. Cereb Cortex. 2019 Apr 1;29(4):1532-46.

25. Hebart MN, Baker Cl. Deconstructing multivariate decoding for the study of brain function. Neurolmage. 2018 Oct 15;180:4-18.

26. Poldrack RA. Inferring Mental States from Neuroimaging Data: From Reverse Inference to Large-Scale Decoding. Neuron. 2011 Dec 8;72(5):692-7.

27. Kriegeskorte N, Mur M, Bandettini P. Representational Similarity Analysis Connecting the Branches of Systems Neuroscience. Front Syst Neurosci [Internet]. 2008 Nov 24 [cited 2020 Apr 1];2. Available from: https://www.ncbi.nlm.nih.gov/pmc/articles/PMC2605405/

28. Parkinson C, Liu S, Wheatley T. A Common Cortical Metric for Spatial, Temporal, and Social Distance. J Neurosci. 2014 Jan 29;34(5):1979-87.

29. Feng $C$, Yan X, Huang W, Han S, Ma Y. Neural representations of the multidimensional self in the cortical midline structures. Neurolmage. $2018 \mathrm{Dec}$ 1;183:291-9.

30. Weaverdyck M, Thornton MA, Tamir D. Stable neural representations of mental states across target people and stimulus modalities [Internet]. PsyArXiv; 2020 [cited 2021 Jan 28]. Available from: https://psyarxiv.com/7axzj/

31. Richardson H, Gweon H, Dodell-Feder D, Malloy C, Pelton H, Keil B, et al. Response patterns in the developing social brain are organized by social and emotion features and disrupted in children diagnosed with autism spectrum disorder. Cortex. 2020 Apr 1;125:12-29.

32. Skerry AE, Saxe R. Neural Representations of Emotion Are Organized around Abstract Event Features. Curr Biol. 2015 Aug 3;25(15):1945-54.

33. Dziura SL, Thompson JC. The Neural Representational Space of Social Memory. Open Mind. 2018 Dec 19;3:1-12. 
34. Pillet I, Op de Beeck H, Lee Masson H. A Comparison of Functional Networks Derived From Representational Similarity, Functional Connectivity, and Univariate Analyses. Front Neurosci [Internet]. 2020 [cited 2020 May 13];13. Available from: https://www.frontiersin.org/articles/10.3389/fnins.2019.01348/full

35. Yarkoni T, Poldrack RA, Nichols TE, Van Essen DC, Wager TD. Large-scale automated synthesis of human functional neuroimaging data. Nat Methods. $2011 ; 8(8): 665$.

36. Alcalá-López D, Smallwood J, Jefferies E, Van Overwalle F, Vogeley K, Mars RB, et al. Computing the Social Brain Connectome Across Systems and States. Cereb Cortex. 2018 Jul 1;28(7):2207-32.

37. Power JD, Mitra A, Laumann TO, Snyder AZ, Schlaggar BL, Petersen SE. Methods to detect, characterize, and remove motion artifact in resting state fMRI. Neurolmage [Internet]. 2014 Jan [cited 2020 Nov 30];84. Available from: https://www.ncbi.nlm.nih.gov/pmc/articles/PMC3849338/

38. R Core Team. R: A Language and Environment for Statistical Computing [Internet]. Vienna, Austria: R Foundation for Statistical Computing; 2020. Available from: https://www.R-project.org/

39. JASP Team. JASP (Version 0.14.1)[Computer software] [Internet]. 2020. Available from: https://jasp-stats.org/

40. Esteban O, Markiewicz CJ, Blair RW, Moodie CA, Isik AI, Erramuzpe A, et al. fMRIPrep: a robust preprocessing pipeline for functional MRI. Nat Methods. 2019 Jan;16(1):111-6.

41. Cox RW. AFNI: Software for Analysis and Visualization of Functional Magnetic Resonance Neuroimages. Comput Biomed Res. 1996 Jun 1;29(3):162-73.

42. Nili H, Wingfield C, Walther A, Su L, Marslen-Wilson W, Kriegeskorte N. A Toolbox for Representational Similarity Analysis. PLOS Comput Biol. 2014 Apr 17;10(4):e1003553.

43. Benjamini Y, Hochberg Y. Controlling the False Discovery Rate: A Practical and Powerful Approach to Multiple Testing. J R Stat Soc Ser B Methodol. 1995;57(1):289-300.

44. Vul E, Harris C, Winkielman P, Pashler H. Puzzlingly High Correlations in fMRI Studies of Emotion, Personality, and Social Cognition. Perspect Psychol Sci. 2009 May 1;4(3):274-90.

45. Oosterhof NN, Connolly AC, Haxby JV. CoSMoMVPA: Multi-Modal Multivariate Pattern Analysis of Neuroimaging Data in Matlab/GNU Octave. Front Neuroinformatics [Internet]. 2016 [cited 2020 Dec 1];10. Available from: https://www.frontiersin.org/articles/10.3389/fninf.2016.00027/full

46. Lage-Castellanos A, Valente G, Formisano E, Martino FD. Methods for computing the maximum performance of computational models of fMRI responses. PLOS Comput Biol. 2019 Mar 8;15(3):e1006397.

47. Fabrigar LR, Wegener DT. Exploratory Factor Analysis. Oxford University Press; 2011. $170 \mathrm{p}$.

48. Ward JH. Hierarchical Grouping to Optimize an Objective Function. J Am Stat Assoc. 1963 Mar 1;58(301):236-44.

49. Aichhorn M, Perner J, Weiss B, Kronbichler M, Staffen W, Ladurner G. Temporo-parietal Junction Activity in Theory-of-Mind Tasks: Falseness, Beliefs, or 
Attention. J Cogn Neurosci. 2009 Jun 1;21(6):1179-92.

50. Andrews-Hanna JR, Saxe R, Yarkoni T. Contributions of episodic retrieval and mentalizing to autobiographical thought: Evidence from functional neuroimaging, resting-state connectivity, and fMRI meta-analyses. Neurolmage. 2014 May 1;91:324-35.

51. Ohnishi T, Moriguchi Y, Matsuda H, Mori T, Hirakata M, Imabayashi E, et al. The neural network for the mirror system and mentalizing in normally developed children: an fMRI study. NeuroReport. 2004 Jun 28;15(9):1483-7.

52. Anzellotti S, Young LL. The Acquisition of Person Knowledge. Annu Rev Psychol. 2020 Jan 4;71(1):613-34.

53. Kovács G. Getting to Know Someone: Familiarity, Person Recognition, and Identification in the Human Brain. J Cogn Neurosci. 2020 Dec;32(12):2205-25.

54. Borghesani V, Narvid J, Battistella G, Shwe W, Watson C, Binney RJ, et al. "Looks familiar, but I do not know who she is": The role of the anterior right temporal lobe in famous face recognition. Cortex. 2019 Jun 1;115:72-85.

55. Kriegeskorte N, Formisano E, Sorger B, Goebel R. Individual faces elicit distinct response patterns in human anterior temporal cortex. Proc Natl Acad Sci. 2007 Dec 18;104(51):20600-5.

56. Arioli M, Gianelli C, Canessa N. Neural representation of social concepts: a coordinate-based meta-analysis of fMRI studies. Brain Imaging Behav [Internet]. 2020 Sep 8 [cited 2020 Sep 17]; Available from: https://doi.org/10.1007/s11682-020-00384-6

57. Skipper LM, Ross LA, Olson IR. Sensory and semantic category subdivisions within the anterior temporal lobes. Neuropsychologia. 2011 Oct 1;49(12):3419-29.

58. Wang $\mathrm{X}$, Wang $\mathrm{B}, \mathrm{Bi} \mathrm{Y}$. Close yet independent: Dissociation of social from valence and abstract semantic dimensions in the left anterior temporal lobe. Hum Brain Mapp. 2019;40(16):4759-76.

59. Zahn R, Moll J, Krueger F, Huey ED, Garrido G, Grafman J. Social concepts are represented in the superior anterior temporal cortex. Proc Natl Acad Sci. 2007 Apr 10;104(15):6430-5.

60. Olson IR, Plotzker A, Ezzyat Y. The Enigmatic temporal pole: a review of findings on social and emotional processing. Brain. 2007 Jul 1;130(7):1718-31.

61. Rice GE, Hoffman P, Binney RJ, Lambon Ralph MA. Concrete versus abstract forms of social concept: an fMRI comparison of knowledge about people versus social terms. Philos Trans R Soc B Biol Sci. 2018 Aug 5;373(1752):20170136.

62. Tsukiura T, Mano Y, Sekiguchi A, Yomogida Y, Hoshi K, Kambara T, et al. Dissociable Roles of the Anterior Temporal Regions in Successful Encoding of Memory for Person Identity Information. J Cogn Neurosci. 2010 Oct;22(10):2226-37.

63. Abel TJ, Rhone AE, Nourski KV, Kawasaki H, Oya H, Griffiths TD, et al. Direct Physiologic Evidence of a Heteromodal Convergence Region for Proper Naming in Human Left Anterior Temporal Lobe. J Neurosci. 2015 Jan 28;35(4):1513-20.

64. Rice GE, Caswell H, Moore P, Hoffman P, Lambon Ralph MA. The Roles of Left Versus Right Anterior Temporal Lobes in Semantic Memory: A Neuropsychological Comparison of Postsurgical Temporal Lobe Epilepsy Patients. Cereb Cortex. 2018 Apr 1;28(4):1487-501. 
65. Tsukiura T, Suzuki C, Shigemune Y, Mochizuki-Kawai H. Differential contributions of the anterior temporal and medial temporal lobe to the retrieval of memory for person identity information. Hum Brain Mapp. 2008;29(12):1343-54.

66. Chang DHF, Ban H, Ikegaya Y, Fujita I, Troje NF. Cortical and subcortical responses to biological motion. Neurolmage. 2018 Jul 1;174:87-96.

67. Kirby LA, Moraczewski D, Warnell K, Velnoskey K, Redcay E. Social network size relates to developmental neural sensitivity to biological motion. Dev Cogn Neurosci. 2018 Apr 1;30:169-77.

68. Saygin AP, Wilson SM, Hagler DJ, Bates E, Sereno MI. Point-Light Biological Motion Perception Activates Human Premotor Cortex. J Neurosci. 2004 Jul 7;24(27):6181-8.

69. Saygin AP. Superior temporal and premotor brain areas necessary for biological motion perception. Brain. 2007 Sep 1;130(9):2452-61.

70. Arioli M, Basso G, Carne I, Poggi P, Canessa N. Increased pSTS activity and decreased pSTS-mPFC connectivity when processing negative social interactions. Behav Brain Res. 2021 Feb 5;399:113027.

71. Isik L, Koldewyn K, Beeler D, Kanwisher N. Perceiving social interactions in the posterior superior temporal sulcus. Proc Natl Acad Sci. 2017 Oct 24;114(43): E9145-52.

72. Walbrin J, Downing P, Koldewyn K. Neural responses to visually observed social interactions. Neuropsychologia. 2018 Apr 1;112:31-9.

73. Pitcher D, Ungerleider LG. Evidence for a Third Visual Pathway Specialized for Social Perception. Trends Cogn Sci. 2021 Feb 1;25(2):100-10.

74. Arioli M, Ricciardi E, Cattaneo Z. Social cognition in the blind brain: A coordinate-based meta-analysis. Hum Brain Mapp [Internet]. [cited 2021 Jan 29];n/a(n/a). Available from: https://onlinelibrary.wiley.com/doi/abs/10.1002/hbm.25289

75. Oba K, Sugiura M, Hanawa S, Suzuki M, Jeong H, Kotozaki Y, et al. Differential roles of amygdala and posterior superior temporal sulcus in social scene understanding. Soc Neurosci. 2020 Sep 2;15(5):516-29.

76. Arioli M, Crespi C, Canessa N. Social Cognition through the Lens of Cognitive and Clinical Neuroscience [Internet]. Vol. 2018, BioMed Research International. Hindawi; 2018 [cited 2021 Jan 29]. p. e4283427. Available from: https://www.hindawi.com/journals/bmri/2018/4283427/

77. Redcay E. The superior temporal sulcus performs a common function for social and speech perception: implications for the emergence of autism. Neurosci Biobehav Rev. 2008;32(1):123-42.

78. Yang DY-J, Rosenblau G, Keifer C, Pelphrey KA. An integrative neural model of social perception, action observation, and theory of mind. Neurosci Biobehav Rev. 2015 Apr 1;51:263-75.

79. Jankowski KF, Moore WE, Merchant JS, Kahn LE, Pfeifer JH. But do you think I'm cool?: Developmental differences in striatal recruitment during direct and reflected social self-evaluations. Dev Cogn Neurosci. 2014 Apr 1;8:40-54.

80. Tan K, Daitch A, Pinheiro-Chagas P, Fox K, Parvizi J, Lieberman M. Human electrocorticography reveals a common neurocognitive pathway for mentalizing about the self and others [Internet]. In Review; 2021 Mar [cited 2021 Mar 15]. 
Available from: https://www.researchsquare.com/article/rs-257986/v1

81. Vanderwal T, Hunyadi E, Grupe DW, Connors CM, Schultz RT. Self, mother and abstract other: An fMRI study of reflective social processing. Neurolmage. $2008 \mathrm{Jul}$ 15;41(4):1437-46.

82. Saxe R, Kanwisher N. People thinking about thinking people: The role of the temporo-parietal junction in "theory of mind." Neurolmage. 2003 Aug 1;19(4):1835-42.

83. Bartra O, McGuire JT, Kable JW. The valuation system: A coordinate-based meta-analysis of BOLD fMRI experiments examining neural correlates of subjective value. Neurolmage. 2013 Aug 1;76:412-27.

84. Nejati V, Salehinejad MA, Nitsche MA. Interaction of the Left Dorsolateral Prefrontal Cortex (I-DLPFC) and Right Orbitofrontal Cortex (OFC) in Hot and Cold Executive Functions: Evidence from Transcranial Direct Current Stimulation (tDCS). Neuroscience. 2018 Jan 15;369:109-23.

85. Chein J, Albert D, O'Brien L, Uckert K, Steinberg L. Peers increase adolescent risk taking by enhancing activity in the brain's reward circuitry. Dev Sci. 2011;14(2):F1-10.

86. Moreira JFG, Telzer EH. Mother still knows best: Maternal influence uniquely modulates adolescent reward sensitivity during risk taking. Dev Sci. 2018;21(1):e12484.

87. Smith AR, Steinberg L, Strang N, Chein J. Age differences in the impact of peers on adolescents' and adults' neural response to reward. Dev Cogn Neurosci. 2015 Feb 1;11:75-82.

88. Pfeifer JH, Masten CL, Borofsky LA, Dapretto M, Fuligni AJ, Lieberman MD. Neural Correlates of Direct and Reflected Self-Appraisals in Adolescents and Adults: When Social Perspective-Taking Informs Self-Perception. Child Dev. 2009;80(4):1016-38.

89. Van der Cruijsen R, Peters S, Zoetendaal KPM, Pfeifer JH, Crone EA. Direct and reflected self-concept show increasing similarity across adolescence: A functional neuroimaging study. Neuropsychologia. 2019 Jun 1;129:407-17. 